\title{
LIQUEFACTION PROCESS OF SAND DURING CYCLIC LOADING
}

\author{
TORU Shibata*, Hiroshi Yukitomo** and Manabu MIYoshi***
}

\begin{abstract}
Both quick cyclic and slow cyclic shear tests are performed on saturated sand to investigate the process of liquefaction under the condition of constant mean principal stress. Special tests with varying amplitude of cyclic shear stress are also performed to estimate the change in excess pore water pressure during irregular cyclic loading.

The pure water pressure increment during each loading cycle may be expressed as a function of the shear stress and the normal stress on the shear plane. This relation is likely to be applicable to the case of irregular cyclic loading which simulates the actual timehistory of shear stress during an earthquake. The number of stress cycles required to cause the initial liquefaction may also be expressed in terms of the ratio of cyclic shear stress to normal stress.

In so far as the sand and glass beads used here are concerned, the pore water pressure tends to increase during unloading and vice versa at the time when initial liquefaction occurs, and the angle of internal friction mobilized at that time has a close relation with the true angle of friction of granular materials.
\end{abstract}

Key words: angle of internal friction, dilatancy, earthquake, effective stress, liquefaction, pore pressure, repeated loading, sand, vibration

IGC: D6/D7

\section{INTRODUCTION}

It is universally known that saturated loose sand exhibits the phenomenon of liquefaction when it is subjected to cyclic loading occurring at relatively short intervals. It may be postulated that the phenomenon is shear failure due to a decrease in internal friction resulting from the cyclic loading, and due to a decrease in effective stress in consequence of rise in pore water pressure.

* Professor, Disaster Prevention Research Institute, Kyoto University, Gokasho, Uji, Kyoto.

** Development Department of Construction Materials, Kawasaki Steel Corporation, Former Assistant of Kyoto University.

*** Graduate Student, Kyoto University.

Written discussions on this paper should be submitted before January 1, 1973. 
To date, numerous researches have been pursued on the liquefaction of sand in connection with the investigation of the possible disastrous effects of earthquakes upon soft ground. A review of the series of achievements by Seed et al. in their researches reveals that the major factors for the development of liquefaction are (1) density of sand, (2) effective overburden pressure on sand, (3) magnitude of cyclic shear stress, and (4) number of stress cycles exerted by an earthquake.

In view of the present status of affairs, the authors attempt to devote a discussion to the excess pore water pressure which builds up in saturated sand when a cyclic shear stress is applied including the case of irregular-amplitude shear stresses which simulates the actual time-history of shear stress during earthquakes. It is further attempted to express the number of stress cycles required to cause liquefaction as the function of shear stress and mean principal stress, based on the understanding that the phenomenon of liquefaction corresponds to the process in which the effective normal stress exerted on the soil element gradually decreases and eventually reaches a critical state.

\section{TEST PROCEDURE AND SAMPLES}

In order to make clear the liquefaction phenomenon of the saturated sand with the aid of a vibratory triaxial apparatus, there are three kinds of loading methods, i.e.,

(1) Only the axial pressure is altered while maintaining the lateral pressure constant.

(2) Both the lateral and axial pressures are changed so as to keep the normal stress $\sigma_{v}$ on the shear plane constant.

(3) Both the lateral and axial pressures are fluctuated, in order to maintain the mean principal stress $\sigma_{m}$ constant.

If the stress conditions affecting the soil element in the ground are such that the normal stress can be regarded constant, then the triaxial apparatus to be employed for this purpose should be so designed as to permit the reproduction of the same stress condition. Because of the complexity of the testing apparatus employed to date, however, no triaxial tests covering the above (2) or (3) have ever been performed on high-speed cyclic loading.

Of course, in working with saturated sands, it may be possible to correct the pore water pressures measured by the method (1) above, as has been attempted by Seed and Lee (1966). But it is most desired that the test be made under the condition of constant $\sigma_{m}$ for detailed study of changes in pore water pressure because of the difficulty of making a perfectly saturated sample of sand.

In the experiments in this paper, both quick cyclic and slow cyclic tests were made. The quick cyclic test as referred to herein would be conducted at a frequency of about 1 $\mathrm{Hz}$, while the slow cyclic test would take several or several tens of minutes to complete one cycle. The quick test was made to meet the condition of constant $\sigma_{m}$ by using vibratory triaxial apparatus (refer to Appendix). The variations of axial pressure $\sigma_{t a}$ and that of lateral pressure $\sigma_{r}$ were controlled so as to maintain the condition of constant $\sigma_{m}$.

Examples of recorded time histories of the lateral pressure $\Delta \sigma_{r}$, axial deviatoric pressure $\Delta \sigma_{d}$, axial strain $\varepsilon_{a}$ and pore water pressure $\Delta u$ are shown in Fig. 1. The curves of $\varepsilon_{a}$ and $\Delta u$ indicate that the quantities of axial deformation and pore water pressure increase 


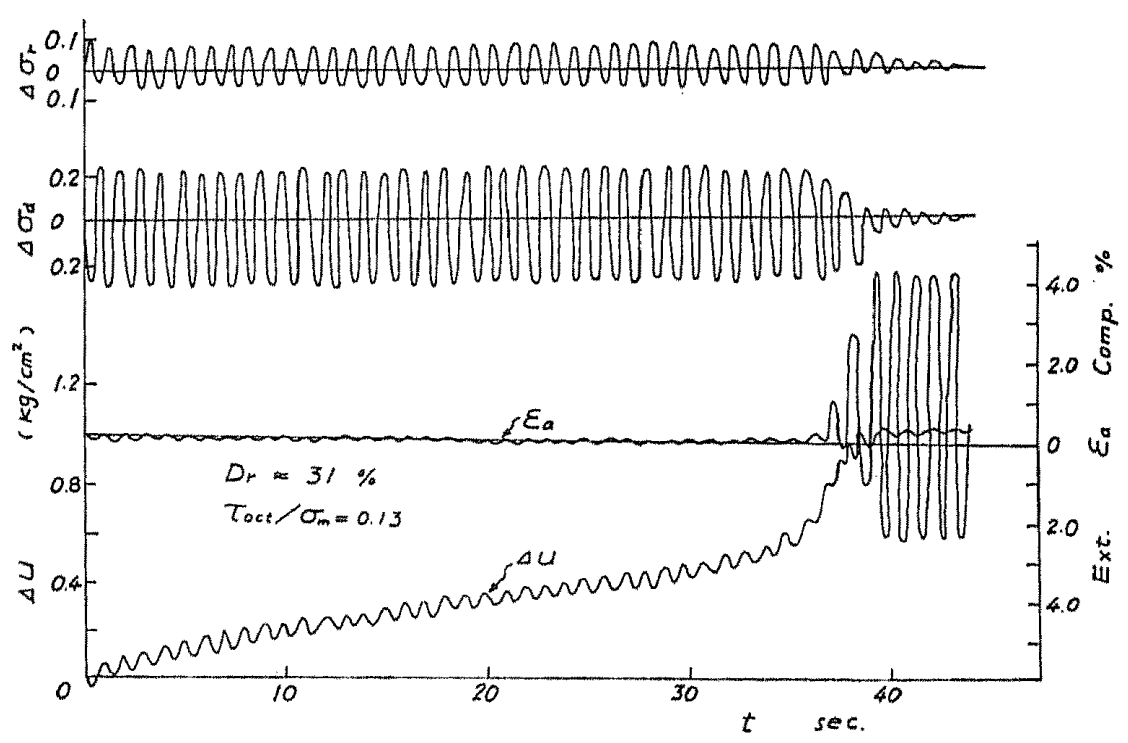

Fig. 1. Record of a typical quick cyclic triaxial test

sharply after $t=35 \mathrm{sec}$. In the stages of partial and complete liquefaction of sand, both $\Delta \sigma_{d}$ and $\Delta \sigma_{r}$ must be decreased as shown in Fig. 1 by mechanical operation, in order to keep the condition of constant $\sigma_{m}$ and to follow the rapid decrease in shearing resistance of sand.

In the case of the slow cyclic test, triaxial and simple shear testing apparatus of the Norwegian Geotechnical Institute type were used. The dimensions of the triaxial specimen are $3.5 \mathrm{~cm}$ in diameter and $8.0 \mathrm{~cm}$ high. For the simple shear test the specimen is $8.0 \mathrm{~cm}$ in diameter and $2.0 \mathrm{~cm}$ high, and the vertical stress was controlled so as to keep the volume of sample constant.

The sample, which had been molded and frozen previously, was thawed and consolidated by applying a lateral pressure of $1.0 \mathrm{~kg} / \mathrm{cm}^{2}$ in the triaxial cell. After the completion of consolidation, a back pressure of $1.0 \mathrm{~kg} / \mathrm{cm}^{2}$ was applied to the sample. Although such a back pressure was added, the sample did not become perfectly saturated, i.e., the measured $B$ values of the coefficient of pore water pressure reached only $0.8-0.9$.

In addition to Toyoura sand on which most tests were conducted, glass beads were used in some tests. The index properties of the samples are given in Table 1.

Table 1. Index properties of samples

\begin{tabular}{cccccc}
\hline Sample & $G_{s}$ & $e_{\max }$ & $e_{\min }$ & $U_{c}$ & $D_{50}(\mathrm{~mm})$ \\
\hline Toyoura sand & 2.65 & 0.95 & 0.63 & 1.5 & 0.16 \\
Glass beads & 2.48 & 0.71 & 0.50 & 1.2 & 0.18 \\
\hline
\end{tabular}

\section{PORE WATER PRESSURE DURING CYCLIC LOADING}

In a typical testing record as shown in Fig. 1, the pore water pressure caused by the cyclic loading increases with time. If the pore water pressure $\Delta u_{m}$ at zero shear stress 


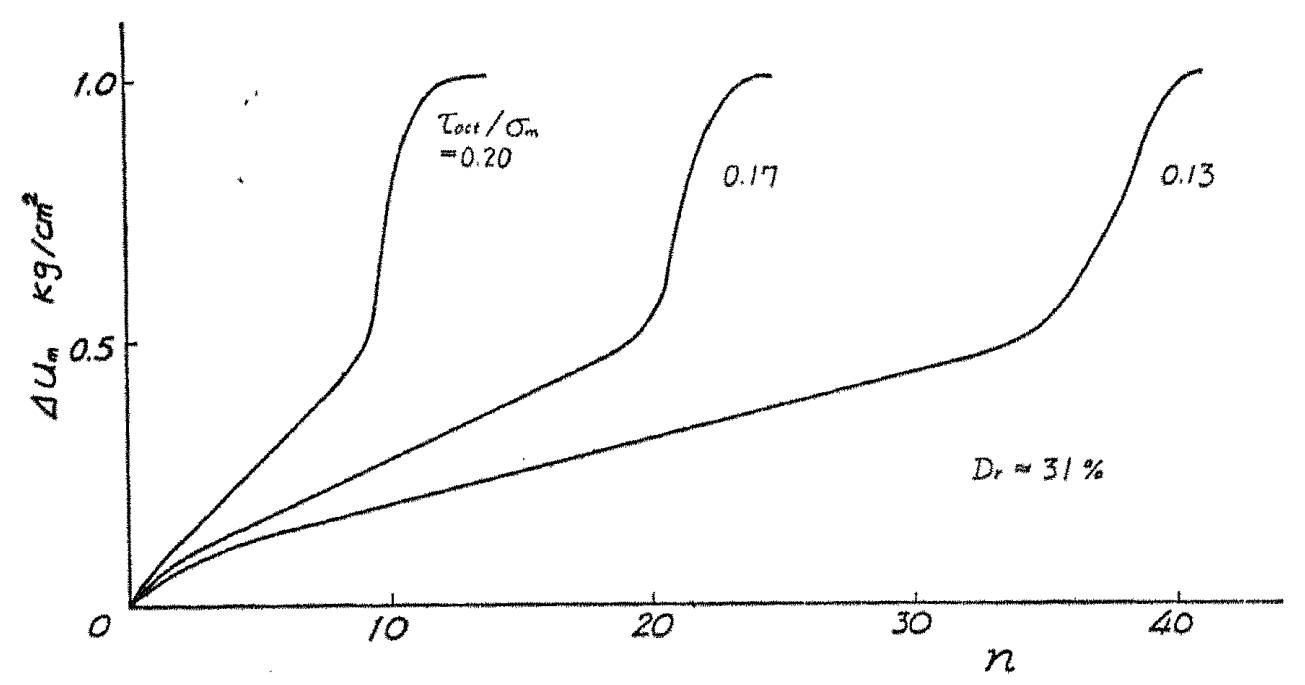

Fig. 2. Pore water pressure change $\Delta u_{m}$ vs. the number of cycles

during each of the stress cycles in Fig. 1 is plotted against the number of cycles $n$, the curve marked as $\tau_{o c t} / \sigma_{m}=0.13$ in Fig. 2 is obtained.

The test results on samples having the same relative density but for different values of the stress ratios $\tau_{o c t} / \sigma_{m}$ are also shown in Fig. 2, where $\tau_{\text {oct }}$ is the octahedral shear stress. It is clear from Fig. 2 that the slope in the middle part of $\Delta u_{m}$ vs. $n$ curve, i.e., the pore water pressure increment during each stress cycle, denoted $\Delta \bar{u}$, increases with an increase in $\tau_{\text {oot }} / \sigma_{m}$.

On the other hand, the change in the pore water pressure in soil caused by a monotonous increase in stress or strain can be given as follows:

$$
\Delta u=\Delta \sigma_{m}+\left(\frac{D}{C}\right) \cdot \tau_{o o t}
$$

where $D$ is the dilatancy coefficient and $C$ is the coefficient of compressibility of soil structure.

If $D / C=\alpha$ and $\Delta \sigma_{m}=0$, the magnitude of $\Delta u$ can be given by the following expression:

$$
\Delta u=\alpha \cdot \tau_{o c t}
$$

The soil element in the ground is assumed to undergo deformation under the plane strain dondition. According to the results of the static shear test in plane strain as performed by Cornforth (1964), the ratio between the intermediate principal stress and the sum of the other principal stresses during shear, $\sigma_{2} /\left(\sigma_{1}+\sigma_{3}\right)$, fall within a range between 0.3 and 0.4 , though variable with the density. In the case of undrained condition, the intermediate principal stress $\sigma_{2}$ becomes $\left(\sigma_{1}+\sigma_{3}\right) / 2$ on the assumption that Poisson's ratio of soil is 0.5 , and consequently the mean principal stress $\sigma_{m}$ is expressed as

$$
\sigma_{m}=\frac{1}{3}\left(\sigma_{1}+\sigma_{2}+\sigma_{3}\right)=\frac{1}{2}\left(\sigma_{1}+\sigma_{3}\right)=\sigma_{v}
$$

If there is no variation in the normal stress $\sigma_{v}$, then $\Delta \sigma_{v}=\Delta \sigma_{m}=0$, and the change in 
pore water pressure $\Delta u$ will be expressed as follows:

$$
\Delta u=\frac{2 \sqrt{2}}{3} \alpha \cdot \tau_{d}
$$

where $\tau_{d}=\left(\sigma_{1}-\sigma_{3}\right) / 2$.

Incidentally, it is clear from the results of the past studies that the value of $\alpha$ in Eq. (2) varies with the magnitude of $\tau_{\text {oct }}$, i.e., the value $\alpha$ increases with an increase in $\tau_{\text {oot }}$. A review of the results of shear tests conducted on sandy soils at a fixed value of $\sigma_{m}$ leads to a conclusion that the relation between $\Delta u / \sigma_{m}$ and $\tau_{\text {ont }} / \sigma_{m}$ can be frequently approximated with a parabolic curve. This simply indicates that $\alpha\left(=\Delta u / \tau_{\text {oot }}\right)$ increases in direct proportion to $\tau_{\text {oct }} / \sigma_{m}$.

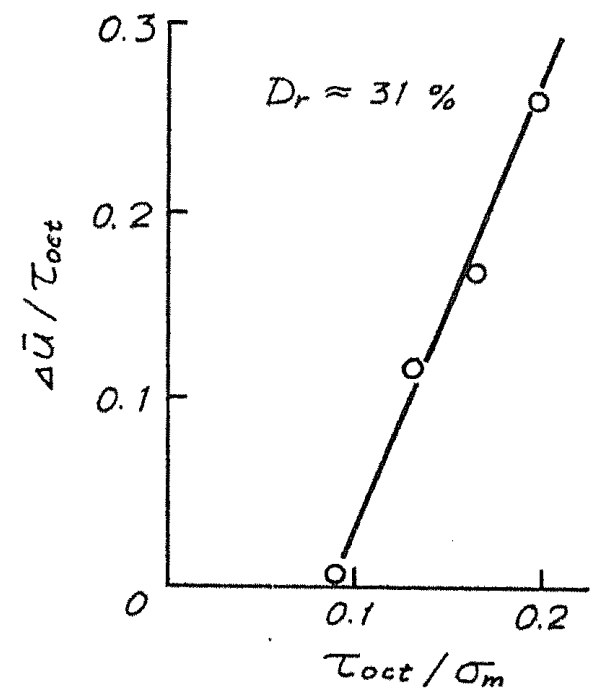

Fig. 3(a). Relationship between pore water pressure increment $\Delta \bar{u}$ during one stress cycle and the stresses $\tau_{o c t}$ and $\sigma_{m}$

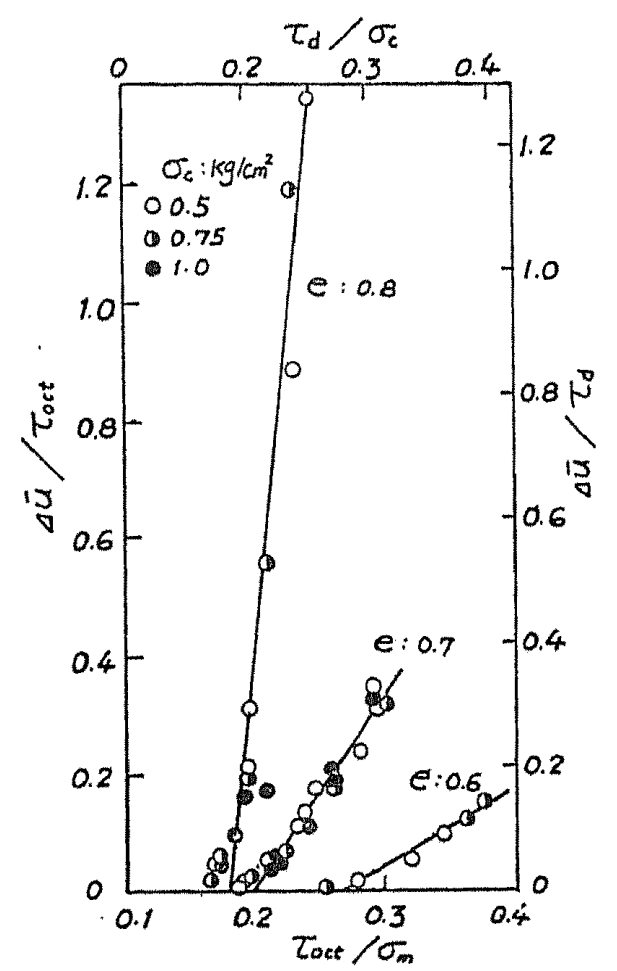

Fig. 3 (b). Relationship between $\Delta \hat{u} / \tau_{o o t}$ and $\tau_{o c t} / \sigma_{m}$ (through analysis of TanimotoSuga's data)

In the above discussion $\Delta u$ was referred to the case where stress increased monotonously, but the pore water pressure increment which accumulats per stress cycle, $\Delta \bar{u}$, during cyclic loading cannot be explained simply by the above equations. Then, a study was made to find the relationship among the following three factors: $\Delta \bar{u}, \sigma_{m}$, and $\tau_{\text {oct }}$. As a result, it is learnt that there is a linear relation when $\Delta \bar{u} / \tau_{\text {oct }}$ is plotted against $\tau_{o c t} / \sigma_{m}$. An example is shown in Fig. 3 (a) for $D_{r} \approx 31 \%$ and this relation can be expressed as follows:

$$
\frac{\Delta \bar{u}}{\tau_{\text {oot }}}=a\left\{\left(\frac{\tau_{\text {oot }}}{\sigma_{m}}\right)-\left(\frac{\tau_{\text {oot }}}{\sigma_{m}}\right)_{0}\right\}
$$


or

$$
\frac{\Delta \bar{u}}{\tau_{d}}=a^{\prime}\left\{\left(\frac{\tau_{d}}{\sigma_{v * 0}}\right)-\left(\frac{\tau_{d}}{\sigma_{v \cdot 0}}\right)\right\}
$$

where $\sigma_{v \cdot o}$ is the initial normal stress on shear plane or equals to $\sigma_{m}$ and $a, a^{\prime}$ are the coefficients dependent upon the kind and density of soil, and $\left(\tau_{o r t} / \sigma_{m}\right)_{c}$ and $\left(\tau_{d} / \sigma_{v \cdot a}\right)_{c}$ denote the critical stress ratios below which liquefaction does not take place.

Fig. 3 (b) was obtained, in such the same way as in Fig. 3 (a), by finding the increments $\Delta \bar{u}$ in pore water pressure for the individual cycles from the test records on a river sand reported by Tanimoto and Suga (1970). From Fig. 3 (b) it will be seen that the postulate of Eq. (3) is approximate and yet is sufficiently valid, and that the coefficient $a$ tends to decrease with the decreasing value of void ratio $e$. The relation in Figs. 3 (a) and (b) will be used for the estimation of pore water pressure in saturated sand during the irregular cyclic loading in the following section.

\section{PORE WATER PRESSURE DURING IRREGULAR CYCLIC LOADING}

The actual time-history of shear stress at any point in a soil deposit during an earthquake will have an irregular form such as that shown in Fig. 4 (a). But the study so far made concerning the liquefaction of saturated sand has been on the assumption that the cyclic stress has a regular form, and there remain problems to be solved before applying the result obtained for such a uniform cyclic stress.

Fig. 4 (a) shows the variation of shear stress with time at the depth of $7.6 \mathrm{~m}$ computed by Seed and Idriss (1967) for the Niigata earthquake of 1964. To assess the liquefaction potential of the sand it is necessary to determine whether the stress variations will induce liquefaction at various depths. For this purpose, Seed and Idriss represented the actual

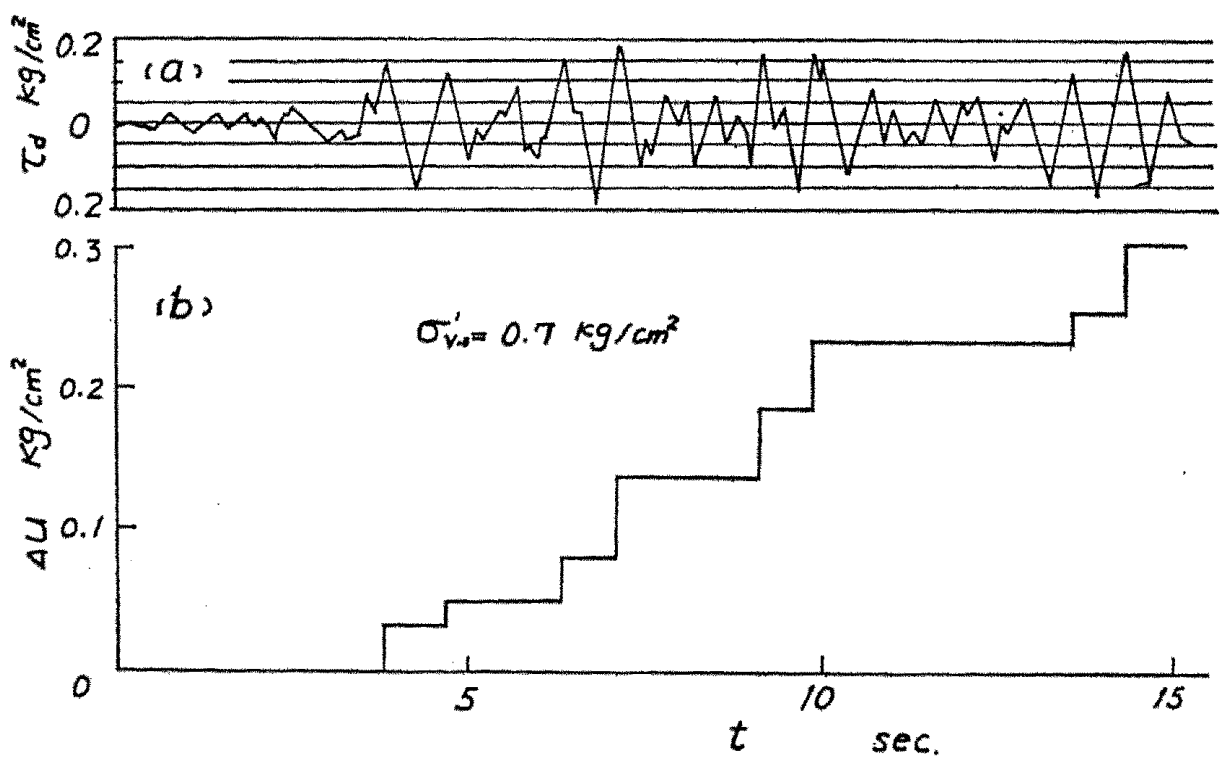

Fig. 4. (a) Time-history of shear stress during earthquake;

(b) Estimated pore water pressure 
stress-time records by an equivalent number of uniform cyclic stress cycles as follows:

In Fig. 4 (a), there are 8 major stress peaks and cycles in the first $15 \mathrm{sec}$ of shaking. The average magnitude of these major stress peaks is $0.16 \mathrm{~kg} / \mathrm{cm}^{2}$. If it is estimated that all the remaining smaller stress cycles are equivalent in their effect to 2 cycles of $0.16 \mathrm{~kg} /$ $\mathrm{cm}^{2}$ magnitude, then the entire stress history for the first $15 \mathrm{sec}$ of shaking can be considered equivalent to 10 cycles of a uniform stress of $0.16 \mathrm{~kg} / \mathrm{cm}^{2}$.

The method adopted by Seed and Idriss is to convert the irregular cyclic stress into an equivalent uniform cyclic stress, which is convenient for practical use but is unclear as to the method of conversion. Therefore, as an approach to the liquefaction problem caused by irregular cyclic stress, a series of special tests was performed in which the amplitude of cyclic shear stress was made to vary under the condition of constant $\sigma_{m}$.

Fig. 5 shows an example of these test results on Toyoura sand at $D_{r}=53 \%$ and outlines the records of variations of lateral pressure $\Delta \sigma_{r}$, axial deviatoric pressure $\Delta \sigma_{d}$, axial strain $\varepsilon_{a}$ and pore water pressure $\Delta u$. In this figure, the sequence of different stress conditions is marked with encircled numbers from (1) to (5). The group of (1), (3), and (5) corresponds to $\tau_{\text {oct }}=0.12 \mathrm{~kg} / \mathrm{cm}^{2}$, and that of (2) and (4) to $\tau_{\text {oct }}=0.17 \mathrm{~kg} / \mathrm{cm}^{2}$. According to Fig. 5, almost the same rate of increase of mean pore water pressure $\Delta u_{m}$ is obtained from the $\Delta u$ vs. $t$ curves for the stress conditions (1), (3), and (5), but for larger stress amplitude of (2) and (4), $\Delta u_{m}$ increases more rapidly. The test results for various magnitudes of cyclic stress amplitude and relative density of sand are given below.

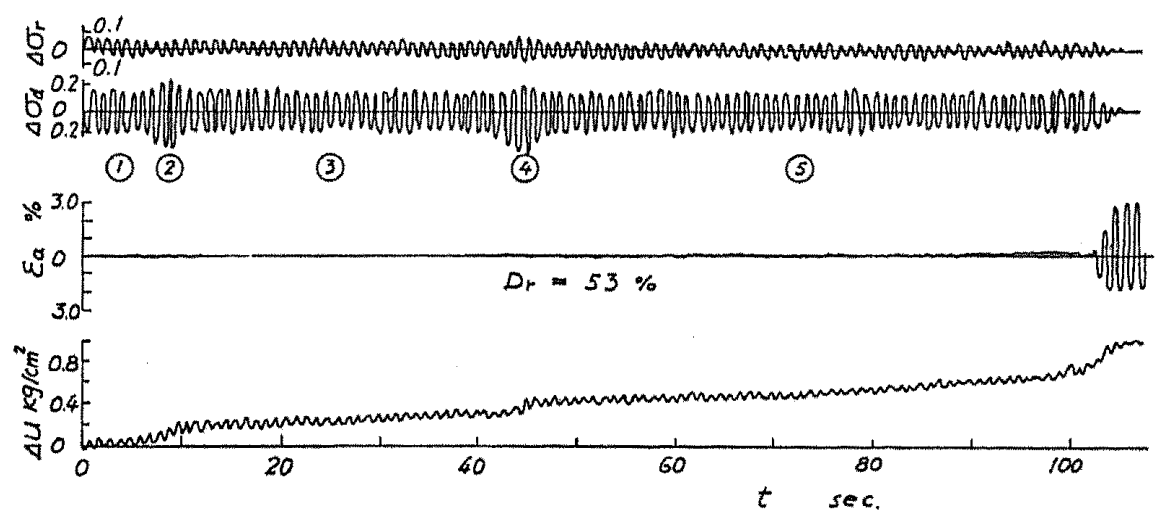

Fig. 5. Record of a typical irregular cyclic loading triaxial test

Fig. 6 (b) shows the record of $\Delta u_{m}$ obtained for the sand at $D_{r}=66 \%$ subjected to the cyclic stress as shown in Fig. 6 (a). In Fig. 6(a), (1), (3), (5), and (7) correspond to $\tau_{o c t}=$ $0.13 \mathrm{~kg} / \mathrm{cm}^{2}$, (2), (4), and (6) to $\tau_{\text {oct }}=0.19 \mathrm{~kg} / \mathrm{cm}^{2}$, and (8) to $\tau_{\text {oct }}=0.24 \mathrm{~kg} / \mathrm{cm}^{2}$.

From this figure the pore water pressure increment $\Delta \bar{u}$ accumulating during one stress cycle may be calculated, as done in the preceding paragraph, and Fig. 7 is obtained by plotting the value of $\Delta \bar{u} / \tau_{o s t}$ against $\tau_{\text {oct }} / \sigma_{m}$. The affixed numbers in Fig. 7 correspond to those in Fig. 6 . According to Fig. 7, a comparison of the magnitude of $\Delta \bar{u} / \tau_{\text {oct }}$ for a fixed value of $\tau_{o c t} / \sigma_{m}$ shows that $\Delta \bar{u} / \tau_{\text {oot }}$ tends to decrease slightly with increasing the sequence number, but this tendency does not seem very marked. 

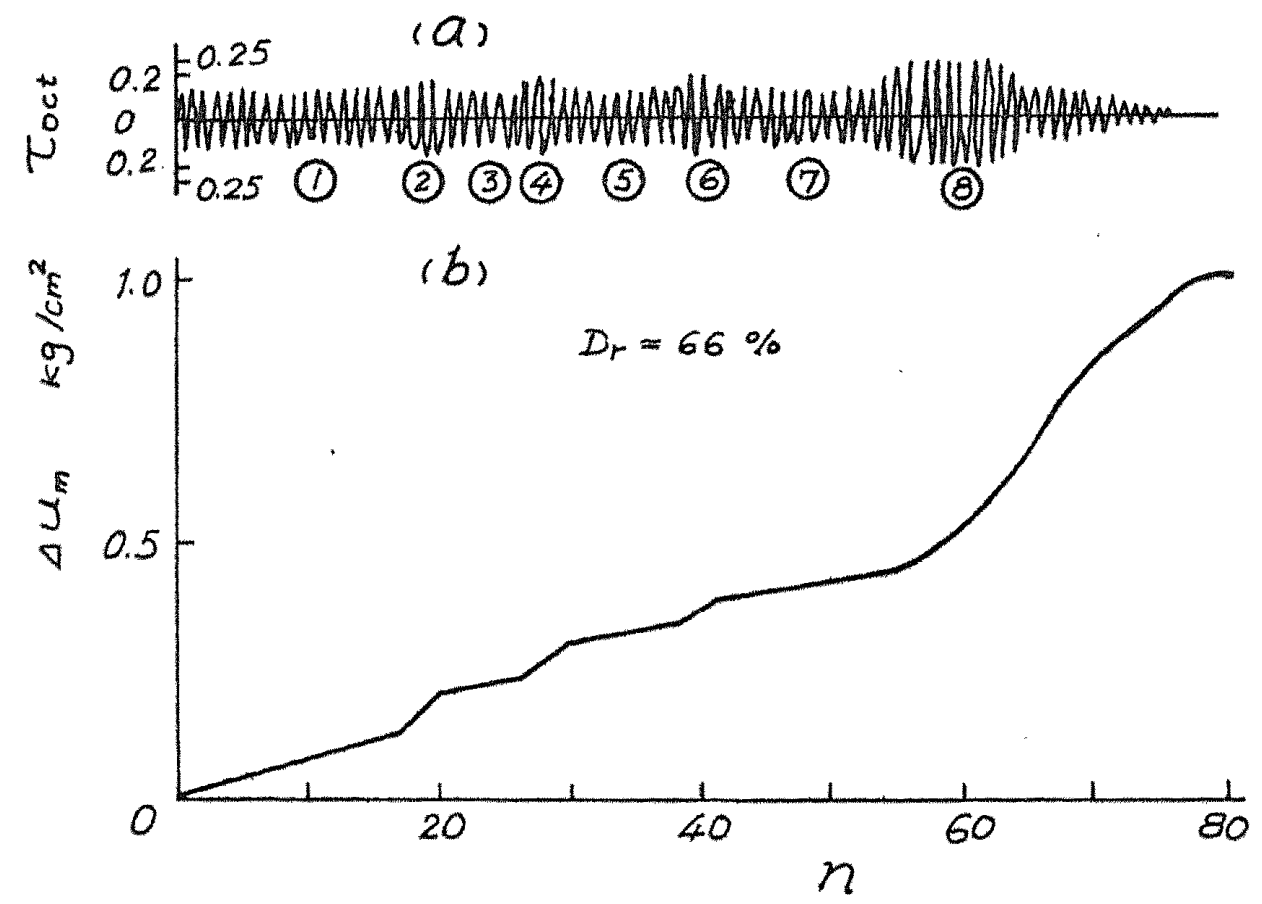

Fig. 6. (a) Applied shear stress; (b) Change in pore water pressure

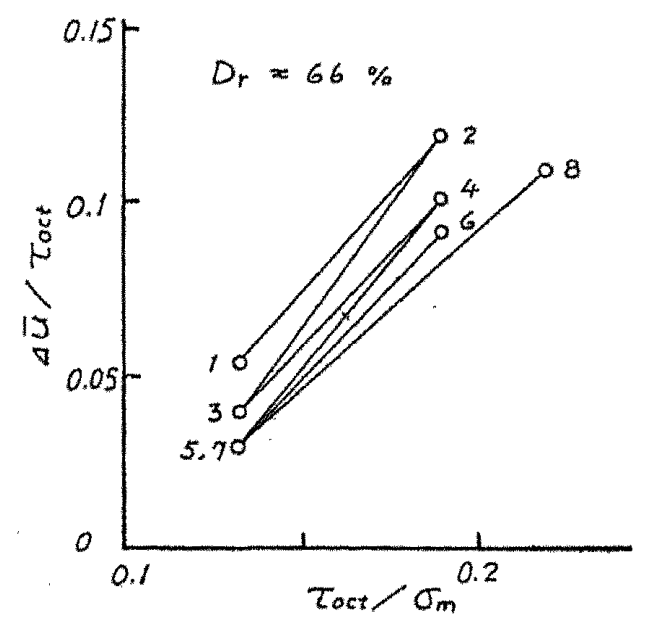

Fig. 7. Relationship between pore water pressure increment $\Delta \tilde{u}$ during one stress cycle and the variables $\tau_{o c t}$ and $\sigma_{m}$ (irregular cyclic loading)

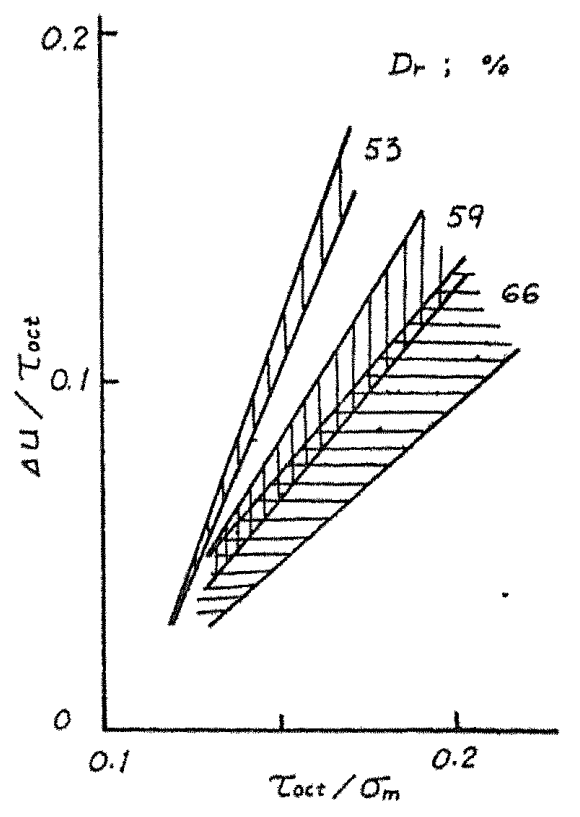

Fig. 8. Relationship between pore water pressure increment $\bar{\Delta} \bar{u}$ during one stress cycle and the variables $\tau_{\text {oot }}$ and $\sigma_{m}$ (irregular cyclic loading) 
If the results of a series of tests on the sand at different densities are plotted in the form of $\Delta \bar{u} / \tau_{\text {oot }}$ vs. $\tau_{\text {oot }} / \sigma_{m}$ relation, Fig. 8 is obtained and this figure corresponds to Fig. 3 mentioned in the preceding section. It is seen from Fig. 8 that the slope of $\Delta \bar{u}$ / $\tau_{\text {oct }}$ vs. $\tau_{\text {oct }} / \sigma_{m}$ lines decreases with an increase in the relative density of sand. If the linear relation between $\Delta \bar{u} / \tau_{\text {oct }}$ and $\tau_{\text {oct }} / \sigma_{m}$ exists as shown in Fig. 8, it becomes possible to estimate the value of pore water pressure during the application of irregular cyclic stresses.

In view of the fact that the grain size distribution curve of Toyoura sand is similar to that of the liquefied sand in Niigata earthquake and that the relative density of the soil layer studied in Fig. 4 (a) is $40 \%$, the relation of $\Delta \bar{u} / \tau_{d}$ vs. $\tau_{d} / \sigma_{v_{* 0}}$ for $D_{r}=40 \%$ has been extrapolated from Fig. 8. In this regard, the calculation was made on the assumption that $\tau_{d}=\left(\sigma_{1}-\sigma_{3}\right) / 2$ and $\sigma_{v \cdot 0}=\sigma_{m}$, as mentioned previously. As a result, the following is obtained:

$$
D_{r}=40 \% ; \quad \frac{\Delta \vec{u}}{\tau_{d}}=2.2\left\{\left(\frac{\tau_{d}}{\sigma_{v \cdot o}}\right)-0.12\right\}
$$

By calculating $\Delta \bar{u} / \tau_{d}$ for various values of $\tau_{d} / \sigma_{v \cdot 0}$, the amount of pore water pressure accumulated with the elapse of time can be estimated. Fig. 4 (b) illustrates the result of such an estimation, and indicates that the pore water pressure of $0.3 \mathrm{~kg} / \mathrm{cm}^{2}$ is generated in the initial shaking period of 15 seconds.

As to the magnitude of equivalent uniform cyclic stress for the number of stress cycles of $10, \tau_{d \cdot 10}=0.15 \mathrm{~kg} / \mathrm{cm}^{2}$ is obtained by substituting $\Delta \bar{u}=0.3 / 10=0.03 \mathrm{~kg} / \mathrm{cm}^{2} / \mathrm{cycle}$ and $\sigma_{v \cdot o}=0.7 \mathrm{~kg} / \mathrm{cm}^{2}$ into the above equation. In the analysis by Seed and Idriss the corresponding value was estimated at $\tau_{d \cdot 10}=0.16 \mathrm{~kg} / \mathrm{cm}^{2}$.

\section{MOBILIZED INTERNAL FRICTION AT INITIAL LIQUEFACTION}

In order to observe the process of liquefaction under the cyclic loading from the viewpoint of effective stress, the vector curve or effective stress path has been drawn. Fig. 9 shows an example of this curve, the relation of $\tau_{d}$ vs. $\sigma_{v}{ }^{\prime}$ at $45^{\circ}$ oblique plane, for a very loose condition of $D_{r}=22 \%$. According to this figure, the excess pore water pressure builds up and, consequently, the effective normal stress $\sigma_{v}{ }^{\prime}$ decreases gradually, and the vector curve progresses toward the left and complete liquefaction occurs when the curve finally reaches the failure line.

In Fig. 9, moreover, the process in which the stress $\sigma_{v}{ }^{\prime}$ decreases almost at equal intervals corresponds to the part of the straight line of the $\Delta u_{m}$ vs. $n$ curve as shown in Fig. 2 . When the vector curve progresses toward the left and the interval between the adjoining peak points on the vector curve becomes larger, the so-called initial liquefaction occurs. The mobilized angle of internal friction $\varphi_{l}^{\prime}$ at the onset of initial liquefaction can be obtained as $\varphi_{l}^{\prime}=25^{\circ}$ as indicated by the dotted line in Fig. 9.

Next, due to the difficulty of examining in detail the soil behavior during the quick cyclic loading in terms of the effective stress, slow triaxial and slow simple shear cyclic tests were carried out. Fig. 10 shows an example of such a triaxial test under the condition of 


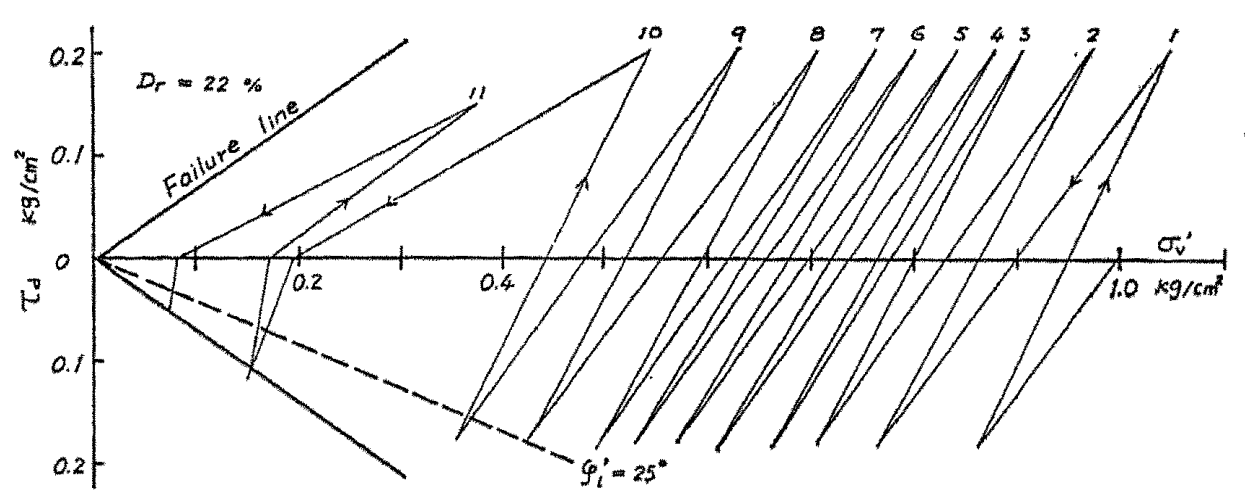

Fig. 9. Vector curve determined from the $\sigma_{n}$-const. quick cyclic triaxial test

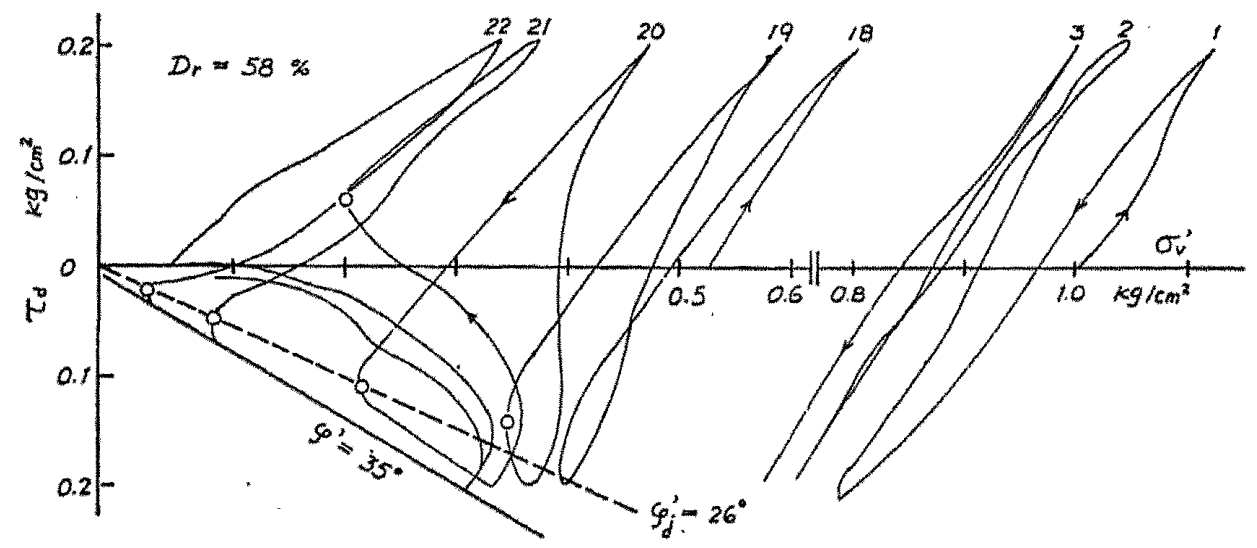

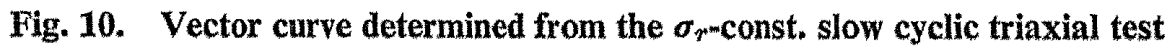

constant lateral pressure. In this figure, when the vector curve reaches the line of $\varphi_{j}{ }^{\prime}=$ $26^{\circ}$ as shown by the dotted line, unusual phenomena called "jumping-out phenomena" by Tanimoto (1970) appear, i.e., the pore water pressure increases during unloading, and vice versa. The points indicated by circles on the vector curve in Fig. 10 corresponds to the occurrence of this phenomenon.

Fig. 11 shows again an example of a vector curve obtained by the slow simple cyclic shear test, and indicates the mobilized angle of internal friction $\varphi_{j}^{\prime}$ being $26^{\circ}$ at the time of the "jumping-out phenomena." In the slow cyclic triaxial and simple shear tests $\varphi_{j}^{\prime}$ was examined on the samples with a wide range in relative density $D_{r}$ of 19 to $67 \%$, and a constant value of $\varphi_{j}^{\prime}=26^{\circ}$ was observed regardless of the kind of tests and the relative density of sand.

Although there is need for further study on the significance of the value of $\varphi_{j}{ }^{\prime}=26^{\circ}$, Murayama and Matsuoka (1970) give an useful suggestion for this problem. They suggest that in the conventional undrained shear test the value of $\tau / \sigma_{v}^{\prime}$ at the time of $\Delta u / \Delta \varepsilon=0$ corresponds to $\tan \varphi_{\mu}$, where $\varphi_{\mu}$ is the true angle of internal friction.

The conventional static undrained shear test with pore water pressure measurement was then performed on Toyoura sand and the change in pore water pressure $\Delta u$ was plotted against the axial strain $\varepsilon$. As a result, the value of 0.47 was obtained for $\tau / \sigma_{v}{ }^{\prime}$ correspond- 


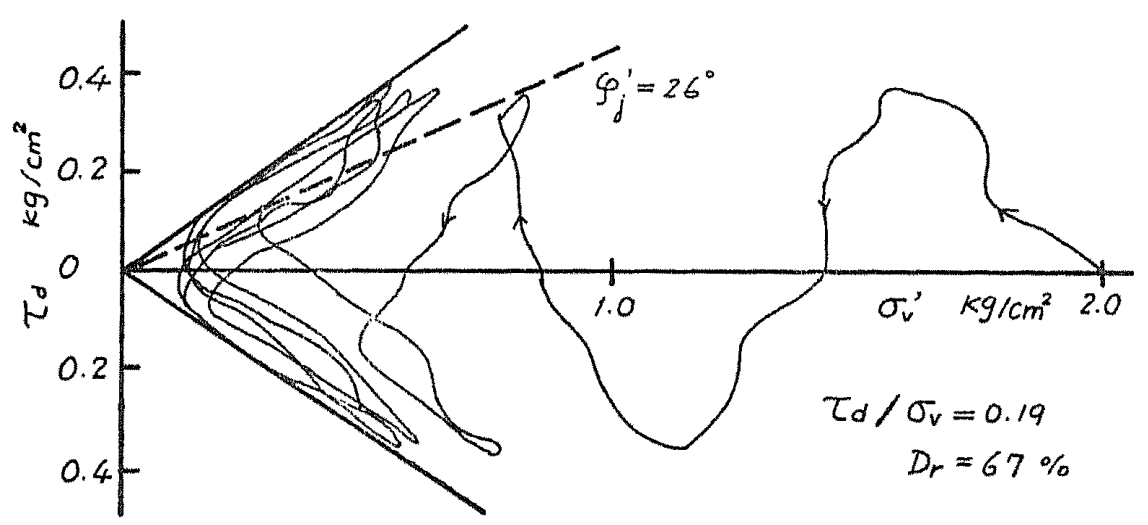

Fig. 11. Vector curve determined from the slow cyclic simple shear test

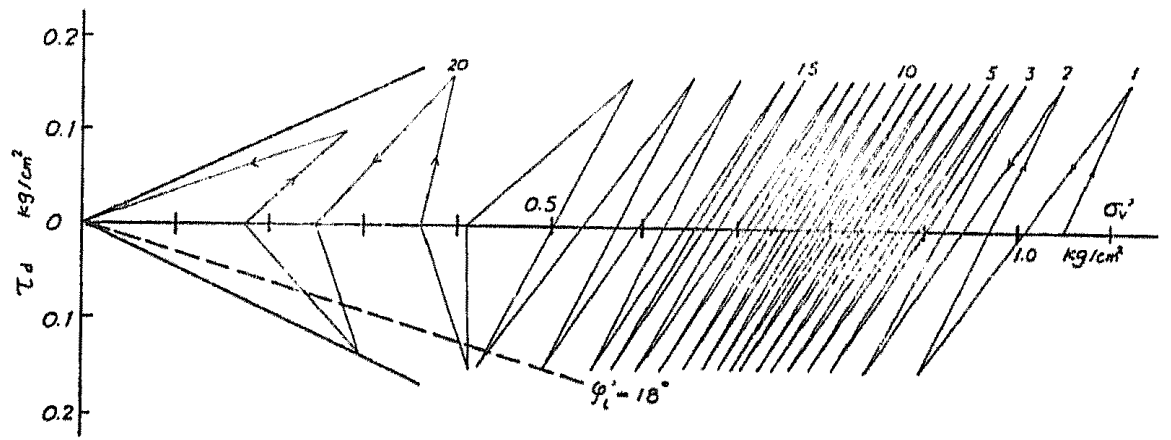

Fig. 12. Vector curve for glass beads (quick cyclic triaxial test)

ing to $\Delta u / \Delta \varepsilon=0$, and the mobilized angle of internal friction was $28^{\circ}$.

On the other hand, by using the $50 \%$ size of Toyoura sand and based on the result of Rowe's paper (1962), the true angle of friction $\varphi_{\mu}$ of $26^{\circ}$ was obtained. From the above slow cyclic or static test, it may be permitted to presume that when the vector curve reaches the line of mobilized internal friction $\varphi_{\mu}$, the "jumping-out phenomenon" takes place.

Now, reverting to the case of quick cyclic test, 8 series of tests were made on Toyoura sand with a range in $D_{r}$ of $22-41 \%$ for measuring the angle of internal friction $\varphi_{l}{ }^{\prime}$ mobilized at the onset of initial liquefaction, and the results showed $\varphi_{l}^{\prime}=23^{\circ}-29^{\circ}$ with the mean value of $26^{\circ}$.

In order to ascertain that $\varphi_{l}{ }^{\prime}$ is found to be close to the true angle of friction $\varphi_{\mu}$, a quick cyclic triaxial test was performed on the glass beads with $\varphi_{\mu}=17^{\circ}$. In Fig. 12 is shown the vector curve thus obtained, and in the case, again, the value corresponding to the mobilized internal friction at the initial liquefaction is very close to $\varphi_{\mu}$.

\section{NUMBER OF STRESS CYCLES TO CAUSE LIQUEFACTION IN TERMS OF STRESS RATIO}

In this section an attempt will be made to express the number of stress cycles $n$ required to cause the initial liquefaction in terms of the stress ratio $\tau_{d} / \sigma_{v^{*} 0}$. For this purpose, the 


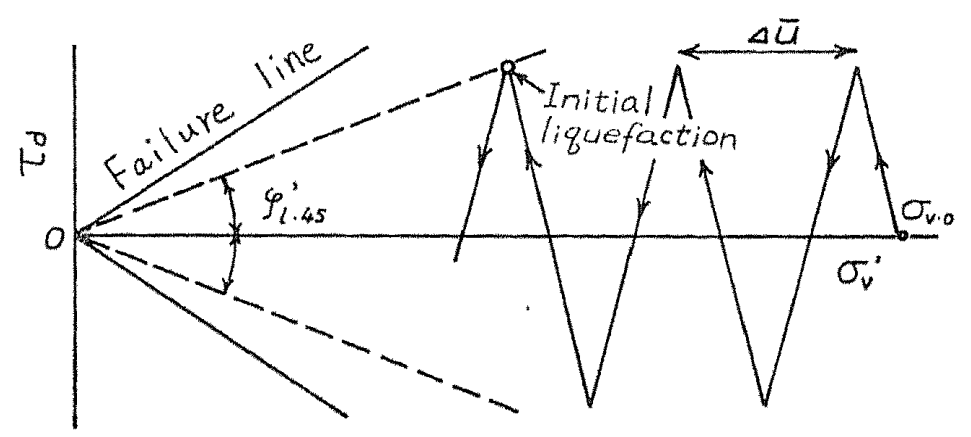

Fig. 13. Schematic vector curve for cyclic loading

idealized vector curve of saturated sand subjected to the cyclic shear stress under undrained condition is shown in Fig. 13. The peaks of the vector curve are plotted at the same intervals and this interval is equal to the pore water pressure increment $\Delta \bar{u}$ accumulated per cycle as given in Eq. (3).

As it is presumed that the initial liquefaction takes place at the time when the vector curve crosses the line passing through the origin with the slope angle of $\varphi_{l \cdot 45}^{\prime}$ the following equation will be obtained from the geometrical relation:

$$
\begin{aligned}
n_{l} & =\left(\sigma_{v \cdot 0}-\tau_{d} \cdot \cot \varphi_{l * 45}^{\prime}\right) / \Delta \bar{u} \\
& =\left\{\left(\frac{\sigma_{v \cdot 0}}{\tau_{d}}\right)-\operatorname{cosec} \varphi_{l}^{\prime}\right\} / a^{\prime}\left\{\left(\frac{\tau_{d}}{\sigma_{v \cdot 0}}\right)-\left(\frac{\tau_{d}}{\sigma_{v \cdot 0}}\right)_{0}\right\}
\end{aligned}
$$

where $\varphi_{l \cdot 45}^{\prime}$ is the mobilized angle of $\varphi_{l}^{\prime}$ on the $45^{\circ}$ oblique plane.

Eq. (4) is what the authors propose here for discussion. It indicates that the number of the stress cycles $n_{l}$ required for the occurrence of the initial liquefaction is the function of the initial normal stress $\sigma_{v \cdot 0}$ and the shear stress $\tau_{d}$, and that the liquefaction becomes more difficult to occur as $\sigma_{v \cdot 0}$ increases and $\tau_{d}$ decreases.

In Fig. 14 the number of stress cycles $n_{i}$ to cause the initial liquefaction is plotted against the relative density $D_{r}$, when $D_{r}$ and $\tau_{o c t} / \sigma_{m}$ are varied in the $\sigma_{m}$-constant quick cyclic tests: the plotted data are grouped in accordance with the value of $\tau_{\text {oct }} / \sigma_{m}$. Other factors being the same, if the value of $\tau_{\text {oot }} / \sigma_{m}$ required to induce liquefaction is assumed to be directly proportional to the relative density of sand, the relation between $\tau_{o c t} / \sigma_{m}$ and $n_{l}$ for any $D_{r}$ may be obtained.

An example of the relation of $\tau_{o c t} / \sigma_{m}$ or $\tau_{d} / \sigma_{v * o}$ vs. $n_{l}$ at a relative density of $31 \%$ thus obtained is shown in Fig. 15, and it may be seen that a unique relationship exists between them in spite of some scatter. The results of calculation of Eq. (4) carried out by using the coefficients of $a^{\prime}$ and $\left(\tau_{d} / \sigma_{v \cdot 0}\right)_{c}$ found from Fig. 3 and the value of $\varphi_{l}^{\prime}$ assumed as $26^{\circ}$ are also shown in Fig. 15 by solid curve. From this figure the curve representing Eq. (4) seems to agree quantitatively with the results of the tests on Toyoura sand with a range in $D_{r}$ of about $20-50 \%$.

Thus, the postulate of Eq. (4) provides a logical explanation of the fact that the phenomenon of liquefaction is more likely to occur as the value of $\tau_{d}$ increases and the value of 


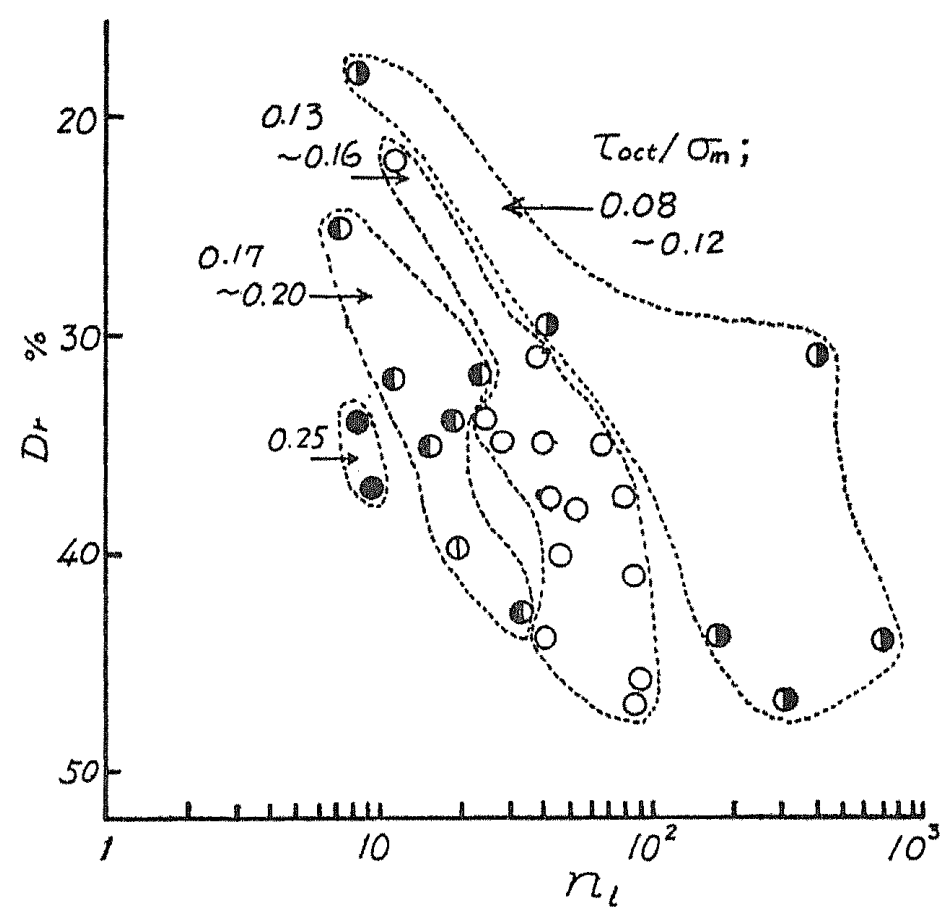

Fig. 14. Relationship between relative density and the number of cycles required to cause liquefaction

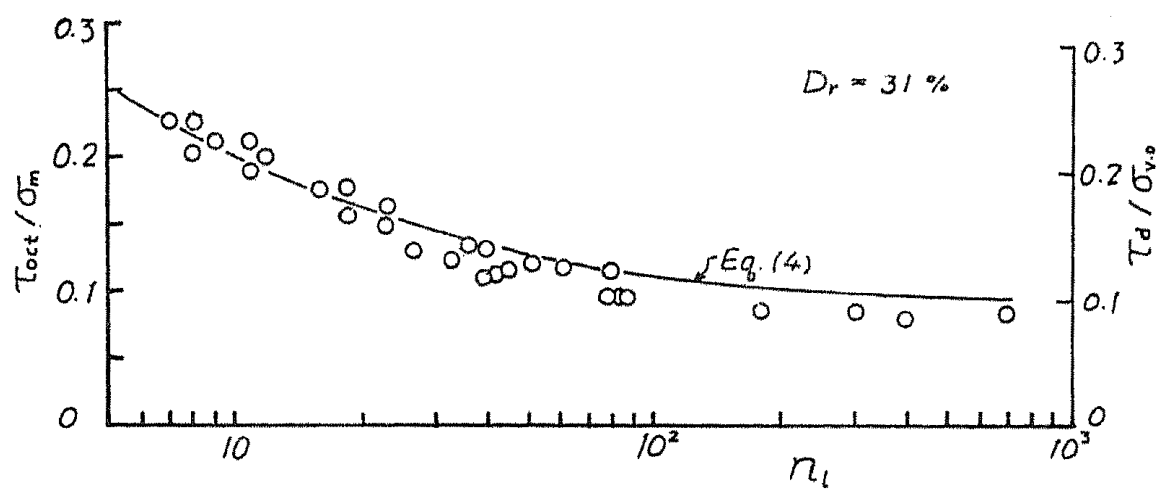

Fig. 15. Relationship between the stress ratio and the number of cycles required to cause liquefaction

$\sigma_{v \cdot o}$ decreases. One factor which governs the phenomenon of liquefaction is the density of sand. As to the effect of this factor, the variation of the two coefficients $a^{\prime}$ and $\left(\tau_{d} / \sigma_{v \cdot{ }^{\circ}}\right)_{c}$ contained in Eq. (4) will suffice for the purpose. Since these coefficients are indices for expressing the volume change under isotropic pressure and dilatancy during shear, they are to be determined by such factors as density, grain size, and grain shape. The significance of these coefficients in the phase of soil mechanics is believed to be elucidated through numerous researches in the future. 


\section{CONCLUSIONS}

A series of quick cyclic shear tests on saturated sands of low density was made in the vibratory triaxial apparatus keeping the mean principal stress constant. In order to study in detail the process of liquefaction from the viewpoint of effective stress, the slow cyclic triaxial and simple shear tests were also made. Moreover, as an approach to the liquefaction of sand caused by irregular cyclic stress, a series of special tests with varying amplitudes of shear stress was performed. The main results based on these tests are as follows:

1. The pore water pressure increment which accumulates during one loading cycle before the onset of initial liquefaction may be expressed as a function of the octahedral shear stress and mean principal stress or that of shear stress and normal stress on the shear plane. This relation is likely to be applicable to the case of irregular stress amplitude which simulates the actual time-history of shear stress during an earthquake.

2. It has been pointed out previously that as the sample approaches the onset of initial liquefaction an unusual change in pore water pressure appears, i.e., the pore water pressure increases during unloading, and vice versa. In so far as Toyoura sand and glass beads used here are concerned, such an unusual change in the pore water pressure appears at the time when initial liquefaction occurs, and the angle of internal friction mobilized at that time has a close relation with the true angle of friction of granular materials.

3. The number of stress cycles required to cause the initial liquefaction may be expressed in terms of the ratio of cyclic shear stress to the mean principal stress. But as for the coefficients included in this expression, there remain uncertainties in their physical meaning for which further study would be required.

\section{ACKNOWLEDGEMENTS}

The authors wish to thank Dr. K. Tanimoto for his kind discussion on their report written in Japanese on the same subject as the present paper, and H. Matsuoka, who gave us a useful suggestion as to the problem of the mobilized internal friction at initial liquefaction.

\section{NOTATION}

The following symbols are used in this paper:

$B=$ coefficient of pore water pressure

$C=$ coefficient of compressibility of soil skeleton

$D=$ coefficient of dilatancy of soil skeleton

$D_{r}=$ relative density

$n=$ number of stress cycles

$n_{l}=$ number of stress cycles required to cause the initial liquefaction

$u=$ excess pore water pressure

$u_{m}=$ pore water pressure corresponding to the zero shear stress during cyclic loading

$\alpha=D / C$ 


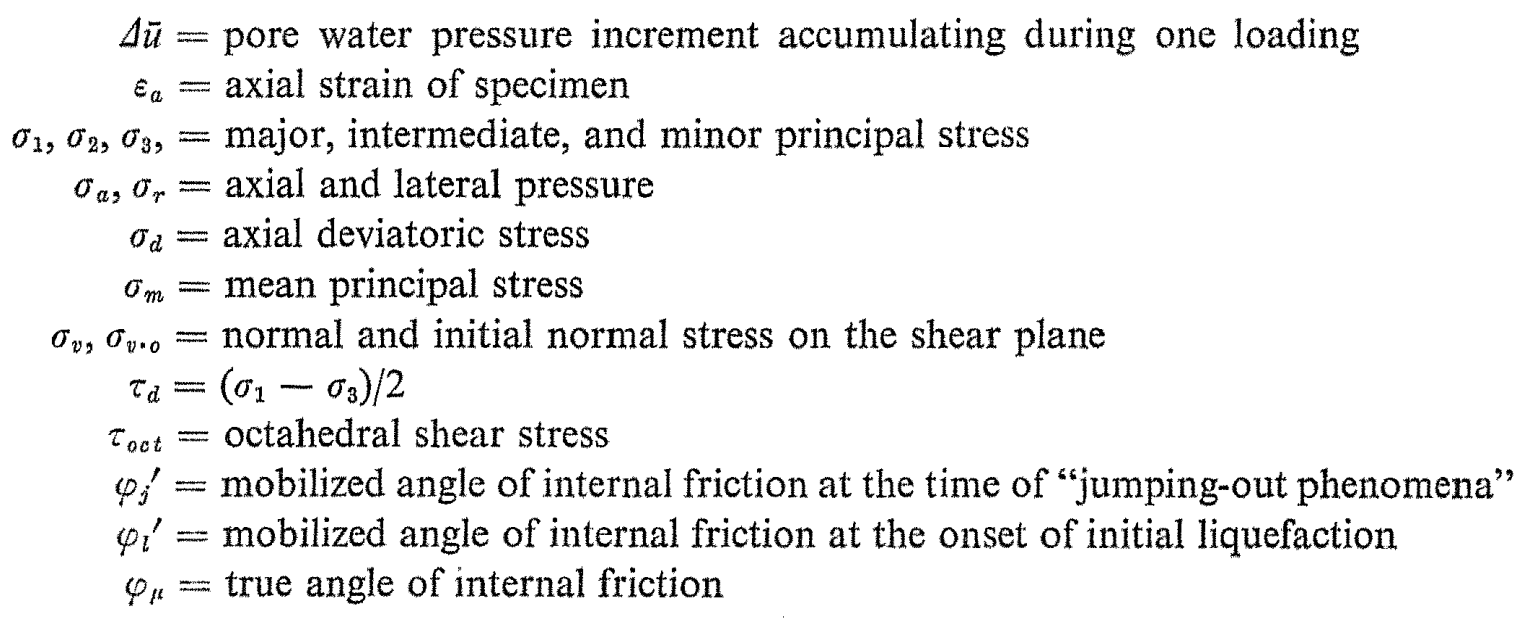

\section{REFERENCES}

Cornforth, D. H. (1964): "Some experiments on the influence of strain conditions on the strength of sand," Geotechnique, Vol. 14, No. 2, pp. 143-167.

Murayama, S. and Matsuoka, H. (1970): "Shearing resistance and structures of granular materials," Proc. 25th Annual Meeting of Japan Society of Civil Engineers, III, pp. 105-108 (in Japanese).

Rowe, P. W. (1962): "The stress-dilatancy relation for static equilibrium of an assembly of particles in contact," Proc. Royal Soc. London, Series A, Vol. 269, pp, 500-527.

Seed, H. B., and Lee, K. L. (1966): "Liquefaction of saturated sands during cyclic loading," Proc. ASCE, Vol, 92, No. SM 6, pp. 105-134.

Seed, H. B. and Idriss, I. M. (1967): "Analysis of soil liquefaction: Niigata earthquake," Proc. ASCE, Vol. 93, No. SM 3, pp. 83-108.

Tanimoto, K. and Suga, Y. (1970): "On the dynamic strength of saturated sands," Proc. 3rd Japan Earthquake Engineering Symp., pp. 191-198.

\section{APPENDIX: TESTING APPARATUS}

A diagrammatic layout of the apparatus is shown in Fig. 16. A belows is inserted in place of the proving-ring of conventional triaxial apparatus, and the water pressure from a vibrating pressure generator is added to the static pressures in the bellows and the triaxial cell. The vibrating pressure generator is composed of two cam cylinders and two bellows in contact with each other. Consequently, vibrating pressure can be added independently to the static axial and lateral pressures. Moreover, the pressure amplitude and frequency of vibration can be increased or decreased continuously over a wide range. The shape of the pulse is a trochoid which is similar to a sinusoidal wave. The phase difference between the axial and lateral vibrating pressures can be changed by the gear fixed to the cam.

The axial pressure and its amplitude are recorded with an electromagnetic oscillograph through a load cell and a dynamic strain meter. The lateral confining pressure and its amplitude are also recorded by the electro-magnetic oscillograph through a pressure head and a dynamic strain meter. The mean value of the axial deformation of the specimen is 


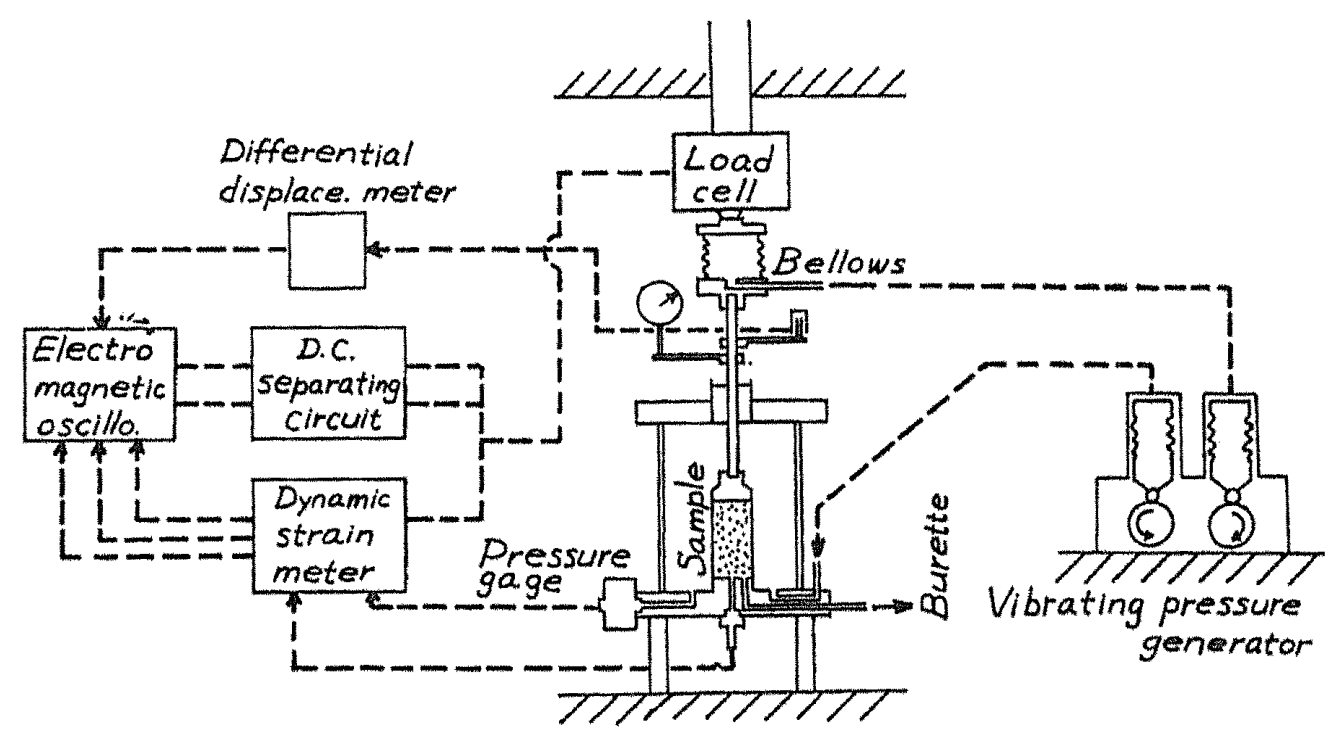

Fig. 16. Layout of the vibrating triaxial apparatus

measured by a dial gage, but the amplitude of deformation at the upper end of the specimer is recorded by the electro-magnetic oscillograph through a differential displacement-meter

(Received September 4, 1971) 